\title{
Alloy characterization of a 7th Century BC archaeological bronze vase - Overcoming patina constraints using Monte Carlo simulations
}

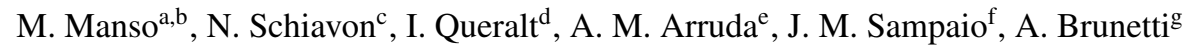 \\ ${ }^{a}$ Laboratório de Instrumentação, Engenharia Biomédica e Física da Radiação (LIBPhys-UNL), Departamento de Física, Faculdade de Ciências \\ e Tecnologias, Universidade Nova de Lisboa, 2829-516 Monte da Caparica, Portugal \\ ${ }^{b}$ Faculdade de Belas-Artes da Universidade de Lisboa, Largo da Academia Nacional de Belas-Artes, 1249-058 Lisboa, Portugal \\ ${ }^{c}$ Hercules Laboratory, University of Évora, Palácio do Vimioso,Largo Marquês de Marialva 8, 7000-809 Évora Portugal \\ ${ }^{d}$ Laboratory of X-ray Analytical Applications, Institute of Earth Sciences Jaume Almera, CSIC, Sole Sabaris s/n, 08028 Barcelona, Spain \\ ${ }^{e}$ Centro de Arqueologia da Universidade de Lisboa, Alameda da Universidade, 1600-214 Lisboa, Portugal

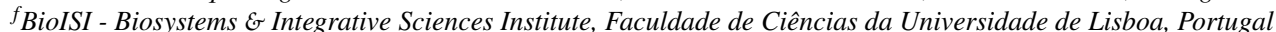 \\ ${ }^{g}$ Department of Political Science and Communication, University of Sassari, Via Piandanna 2, 07100 Sassari, Italy
}

\begin{abstract}
In this work we evaluate the composition of a bronze alloy using X-ray fluorescence spectrometry (XRF) and Monte Carlo (MC) simulations. For this purpose, a 7th Century BC archaeological vase from the SW Iberian Peninsula, displaying a well formed corrosion patina was analysed by means of a portable X-ray fluorescence spectrometer. Realistic MC simulations of the experimental setup were performed with the XRMC code package which is based on an intensive use of variance-reduction techniques and uses XRAYLIB a constantly updated X-ray library of atomic data. A single layer model was applied for simulating XRF of polished/pristine bronze whereas a two or three layers model was developed for bronze covered respectively by a corrosion patina alone or coupled with a superficial soil derived crust. These simulations took into account corrosion (cerussite $\left(\mathrm{PbCO}_{3}\right)$, cuprite $\left(\mathrm{Cu}_{2} \mathrm{O}\right)$, malachite $\left(\mathrm{Cu}_{2} \mathrm{CO}_{3}(\mathrm{OH})_{2}\right)$, litharge $(\mathrm{PbO}))$ and soil derived products (goethite $(\mathrm{FeO}(\mathrm{OH}))$ and quartz $\left.\left(\mathrm{SiO}_{2}\right)\right)$ identified by means of X-ray diffraction and Raman micro analytical techniques. Results confirm previous research indicating that the XRF/Monte Carlo protocol is well suited when a two-layered model is considered, whereas in areas where the patina+soil derived products crust is too thick, X-rays from the alloy substrate are not able to exit the sample. Quantitative results based on $\mathrm{MC}$ simulations indicate that the vase is made of a lead-bronze alloy: $\mathrm{Mn}(0.2 \%), \mathrm{Fe}(1.0 \%), \mathrm{Cu}(81.8 \%), \mathrm{As}(0.5 \%)$, $\mathrm{Ag}(0.6 \%)$, Sn (8.0\%) and $\mathrm{Pb}(8.0 \%)$.
\end{abstract}

Keywords: XRF, Monte Carlo simulations, Archaeological bronzes

\section{Introduction}

The identification of ancient technologies of production from the determination of chemical composition and morphology of archaeological objects often allows a clear attribution to a specific geographical region, as well as, an elucidation of the date of its manufacture, leading to a better understanding of ancient cultures. Furthermore, chemical characterization of archaeological objects may also contribute to a better evaluation of their surface deterioration and to determine the authenticity of the artifacts [1].

$\mathrm{X}$-ray fluorescence spectrometry (XRF) is a simple and fast technique that allows elemental determination

Email address: brunetti@uniss .it (A. Brunetti) and quantification $(\mathrm{Z}>12)$ of metal alloys in a nondestructive way. This technique has been widely used providing new insights concerning the evolution of the use of metals [2, 3, 4, 5] and their provenance [6, 7] through the identification of the component materials.

Advances in technology contributed to the miniaturization of X-rays detectors and tubes enabling the implementation of portable and handheld spectrometers able to provide in-situ measurements [8]. Realistic Monte Carlo (MC) simulations of experimental setups have been developed, in recent years, as a quantification tool able to take into account the roughness of the surface [7, 9].

Alloyed objects suffer from atmosferic corrosion which leads to the formation of various chemical compounds such as oxides, carbonates, sulfides, or sulfates 
on the metal surface. This corrosion layer is known as a patina. The presence of patinas in alloyed objects is often a constraint for its accurate characterization, as the analysis by XRF determines the composition of this superficial corrosion layer formed on the object's surface and not the composition of the underlying material. For an accurate chemical characterization the patina must be removed, which is frequently not allowed. The difficulties in analyzing archeological bronzes because of the alloy's heterogeneity and surface patina, as well as the possibilities and limits of this analytical method have been emphasized in the literature as a topical issue in archaeometry and conservation science $[6,10]$.

In this work we combine XRF spectrometry and MC simulations to evaluate the composition of a bronze alloy with no need for patina removal. An archaeological bronze vase from an important 7th century BC Phoenician settlement (Alcácer do Sal) in the SW part of the Iberian Peninsula was analyzed using a portable XRF spectrometer. MC simulations of the experimental setup were performed with the XRMC code package $[11,12]$

\section{Material and Methods}

The archaeological bronze vase selected for the analytical protocol was found in the 7th century BC necropolis of Senhor dos Mártires near Alcácer do Sal in Southern Portugal, a well known Phoenician site in the protohistory of the Iberian Peninsula [13, 14]. It was casted using the lost-wax technique and has been interpreted as having a ritual funerary use related to libation and purification practices.

Determination of corrosion and soil derived products was carried out by X-ray diffraction (XRD). Raman microscopy was used to study these alterations when they were found to be amorphous [15]. For this purpose samples were collected from the surface, and for the alloy determination, XRF analyses were carried out on the vase.

\section{1. $X R D$}

The mineralogical composition of the bronze corrosion patina was determined by XRD, using a commercial D2-Phaser, Bruker/AXS diffractometer with a $\mathrm{Cu}$ $\mathrm{K} \alpha$ source operating at $30 \mathrm{kV}$ and $10 \mathrm{~mA}$ and a lynxeye detector. The samples scraped from the vase were deposited onto a flat glassy support and irradiated through a $0.6 \mathrm{~mm}$ slit. The angular range $(2 \theta)$ was scanned from $6^{\circ}$ to $80^{\circ}$ at a step size of $0.02^{\circ}$ with a counting time of $0.5 \mathrm{~s} / \mathrm{step}$. Evaluation of X-ray diffractograms was

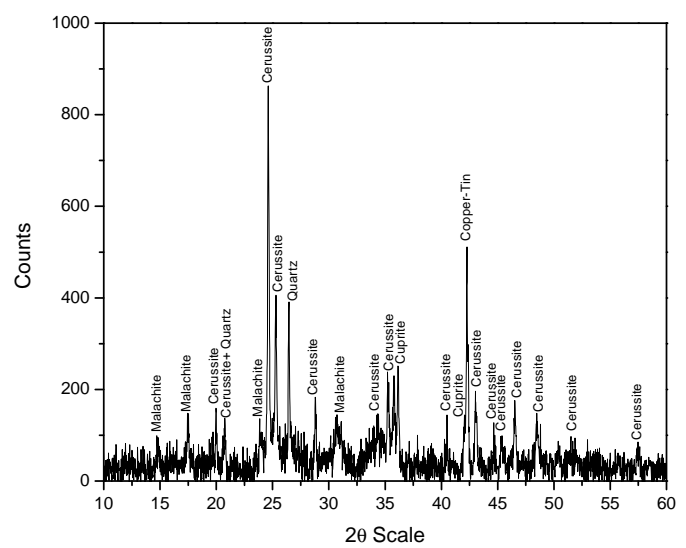

Figure 1: XRD diffractogram of patina and soil derived products identified on the vase.

made by using the routines of the Diffrac software package (BRUKER/AXS GmbH, Germany) and the attached specific PDF database files.

\subsection{Raman}

Raman analyses were undertaken using a HoribaJobin Yvon XploRA confocal spectrometer, operating at wavelength of $785 \mathrm{~nm}$, with maximum incident power of $0.2 \mathrm{~mW}$. Using a 100 magnification objective with a pinhole of $500 \mu \mathrm{m}$ and an entrance slit of $100 \mu \mathrm{m}$, the scattered light collected by the objective was dispersed onto the air-cooled CCD array of an Andor iDus detector by a 1200 lines/mm grating. Raman microscopy performed in a range of $100-1200 \mathrm{~cm}^{-1}$. Spectra deconvolution was performed using LabSpec (V5.78). The identification of corrosion and soil derived products was made in good agreement with literature [16] and Spectral ID ${ }^{T M}$.

\subsection{EDXRF and MC simulations}

The portable spectrometer consists of an Amptek Mini X X-ray tube equipped with a silver anode (50 $\mathrm{kV}, 80 \mu \mathrm{A})$ and an Amptek X-123SDD detector with a resolution of $140 \mathrm{eV} \mathrm{FWHM} \mathrm{at} \mathrm{Mn} \mathrm{K} \alpha(5.9 \mathrm{keV})$. Due to the morphology of the samples, the detector was placed orthogonally to the sample surface while the Xray tube was at $20^{\circ}$ with respect to the normal at the surface. Setup was calibrated using certificated bronze sample [17]. All measurements were carried out under ambient air.

An innovative reverse MC simulation quantification approach was used. In this approach XRF spectra were 
simulated using XRMC code package, which is a fast $\mathrm{X}$-ray interaction simulation code. The code uses a set of files describing both the experimental setup and the composition and structure of the sample. The initial estimate is based on the expected composition of the sample. In the case of the bronze layer only its composition is required whereas in the presence of a patina layer both compositon and thickness are needed. Both compositions as well as the patina thickness are adjusted up to the best fit is obtained for the peak part and background. MC simulations took into account patina and soil derived products composition.

The XRMC code is based on an intensive use of variance-reduction techniques and uses XRAYLIB a constantly updated X-ray library of atomic data [18]. Besides the speed it has, to the best of our knowledge, another unique capability: simulation of arbitrarily rough surfaces [19]. Currently the fitting is user driven but an automatic procedure is being developed, although due to the large number of parameters involved a full automatization will be hard to achieve.

The surface of the artefact shows strong effects of corrosion as well as the presence of a crust layer of soil derived products. The first step was to observe at the microscope the state of the surface in order to choose the optimal experimental setup. A model of the roughness was implemented in the MC code and the optimal $\mathrm{X}$-ray beam size was defined to be $3 \mathrm{~mm}$ wide. The detector and the X-ray tube were modelled following the same geometry of the experimental layout. The distance of X-ray tube from the surface of the object was about $2 \mathrm{~cm}$, while the detector was placed $3 \mathrm{~cm}$ from the surface. The X-ray tube worked at $40 \mathrm{kV}$ and the current was chosen according to the flux reaching the detector. Several points have been analyzed. Here we report the results obtained taking the X-ray spectra at patinated points as well as at cleaned ones. The goal is to demonstrate that the MC simulation is able to give a correct estimative of the bronze composition even in the presence of a corrosion+crust layer. The geometry adopted for the structure of the artefacts depends on the surface state: a single layer geometry for the cleaned surface, a two layers geometry for surfaces with patina (corrosion) and finally a three layers geometry when a crust layer (soil derived products) is also observed. The layers thicknesses as well as the elemental weight percentage have been changed in the simulations to fit the measured spectra.

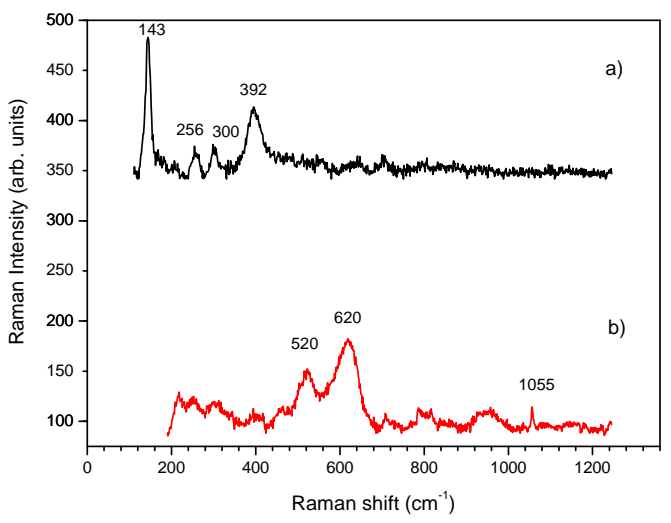

Figure 2: Raman spectra of patina and soil derived products identified on the vase: a) litharge and goethite, b) cuprite and cerussite.

\section{Results and discussion}

XRD analysis revealed that the main crystalline phases of the patina are cerussite $\left(\mathrm{PbCO}_{3}\right)$, cuprite $\left(\mathrm{Cu}_{2} \mathrm{O}\right)$, malachite $\left(\mathrm{Cu}_{2} \mathrm{CO}_{3}(\mathrm{OH})_{2}\right)$ and quartz $\left(\mathrm{SiO}_{2}\right)$. Additional peaks can be attributed to the bronze support (Fig. 1). Raman analysis confirmed the presence of cerussite through the identification of the $1055 \mathrm{~cm}^{-1}$ band. The large band spreading from 490 to $660 \mathrm{~cm}^{-1}$ with two maxima located at 520 and $620 \mathrm{~cm}^{-1}$ has been reported as a characteristic of defective cuprite [20]. The band at $143 \mathrm{~cm}^{-1}$ is ascribed to litharge (PbO) (Fig. 2). Furthermore, goethite was also identified through the presence of the characteristic bands at 256,300 , and $392 \mathrm{~cm}^{-1}$. Goethite and quartz are not the result of corrosion, but instead a soil derived products.

$\mathrm{X}$-ray fluorescence spectra obtained from patina and cleaned region are shown in Fig. 3. The main changes that can be observed are in the peak relative width and in the intensity of the background, which is a marker for the presence of a patina layer. When a patina layer is present the intensity of the background raise. This can be noticed, in the case of bronze, with the level of the background with respect to the $\mathrm{Cu}-\mathrm{K}$ peak baseline. Results confirm previuos research [10] indicating that the XRF/Monte Carlo protocol is well suited when a two-layered model is considered, whereas in areas where the patina plus the crust layer is too thick, X-rays from the alloy substrate are not able to exit the sample. Hence a two layers model was adopted where the outer layer is formed by oxide and other components identified by XRD and Raman spectroscopy, and the inner layer formed by pure bronze whereas for polished areas 
a single layer model was applied. MC simulations have been performed and compared to the spectra obtained at different points on the surface. The surface state at these points is clearly different and the patina thickness appears to be high. The best fit was obtained for the spectra where the intensity of copper was about $60 \%$ lower than in the spectra measured in the regions without patina, meaning there is a thick layer of patina. The spectrum obtained from the simulation of the vase surface is reported in Fig. 4 a) superimposed to the experimental spectrum. In this case the simulation gives the following weight percentage: $\mathrm{Mn}(0.2 \%), \mathrm{Fe}(1.0 \%), \mathrm{Cu}$ (81.8\%), As (0.5\%), Ag (0.6\%), Sn (8\%) and $\mathrm{Pb}(8.0 \%)$. In Fig. 4 b) the spectrum simulated for the clean surface is shown superimposed to the experimental spectrum. In this case the weight percentage obtained are: $\mathrm{Mn}$ $(0.2 \%), \mathrm{Fe}(0.8 \%), \mathrm{Cu}(82.2 \%)$, As $(0.5 \%), \mathrm{Ag}(0.3 \%)$, $\mathrm{Sn}(7 \%)$ and $\mathrm{Pb}(9.0 \%)$. These values are consistent with the ones obtained when the simulation included the outer patina layer. The standard deviation value is a conservative estimation taking account of the errors on the tabulated cross section, especially when L lines are used. Usually the error estimated against reference sample is contained within $1-2 \%$. In the current study, it was estimated at $10 \%$ for most of the elements with the exception of $\mathrm{Ag}$ where a difference of $50 \%$ was found. This could be due to surface accumulation of this chemical element in the oxide layer. However, from the point of view of the bronze composition these level of concentration of a chemical element are not significative. Similar results were obtained in another point of analysis: $\mathrm{Mn}(0.2 \%), \mathrm{Fe}(1.0 \%), \mathrm{Cu}(81.8 \%)$, As $(0.5 \%), \mathrm{Ag}$ $(0.6 \%), \mathrm{Sn}(8 \%)$ and $\mathrm{Pb}(8.0 \%)$ on the vase surface; $\mathrm{Mn}$ $(0.3 \%), \mathrm{Fe}(0.7 \%), \mathrm{Cu}(81.8 \%)$, As $(0.3 \%), \mathrm{Ag}(0.3 \%)$, $\mathrm{Sn}(7 \%)$ and $\mathrm{Pb}(9.0 \%)$ on the clean surface.

\section{Conclusions}

When dealing with unique and invaluable artefacts, the techniques applied have to be non-destructive for the object under investigation. A practical attractive aspect of modern analytical technology is the production of mobile XRF spectrometer that can be transported and operated at the archaeological site or museum. Nevertheless, bronze alloys tend to suffer corrosion during burial and so the surface layer may be thick compared with the penetration depth of the X-rays. In this work we overcome this constraint, by combining an experimental in situ methodology with, accurate, MC simulations of the experimental setup that takes into account sample roughness. The use of XRMC code to simulate

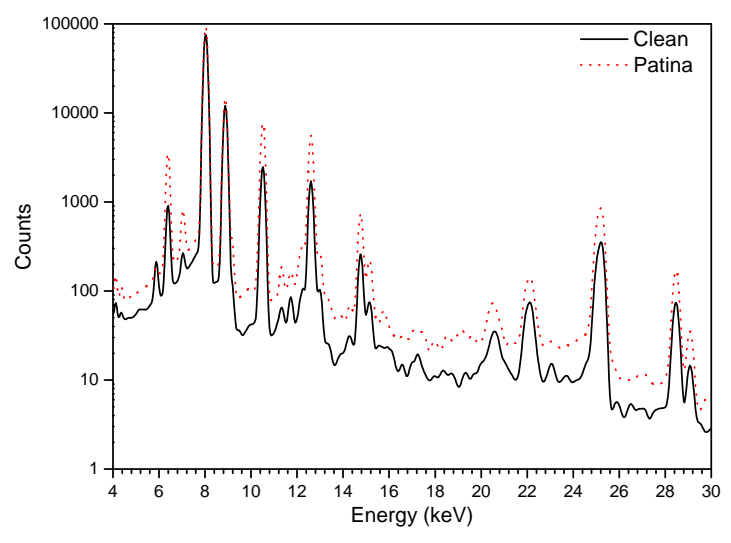

Figure 3: EDXRF spectra obtained before and after patina removal.

the experimental XRF spectra, including the composition of corrosion products obtained through XRD and Raman techniques, allowed the characterization of the alloy's vase as a leaded bronze. The $\sim 10 \%$ of lead concentration is an indication that this element was added to the vase to prevent galvanic corrosion and eventually to making it heavy and thus more stable [2]. Furthermore the use of MC simulations combined with XRF analysis is a valuable tool that favours patina sampling of the areas of analysis over patina removal, which is of most importance when dealing with archaeological objects.

\section{Aknowledgments}

M. Manso acknowledges the support of the Portuguese Foundation for Science and Technology (FCT) for the grant Ref. SFRH/BPD/70031/2010. J. M. Sampaio acknowledges also the financial support of FCT through the research project Ref. EXPL/FISATO/0776/2012.

\section{References}

[1] A. Domenech-Carbo, M.-T. Domenech-Carbo, V. Costa, Application of instrumental methods in the analysis of historic, artistic and archaeological objects, in: Electrochemical Methods in Archaeometry, Conservation and Restoration, Springer Berlin Heidelberg, 2009.

[2] V. K. Gouda, G. I. Youssef, N. A. Abdel Ghany, Characterization of Egyptian bronze archaeological artifacts, Surf. Interface Anal. 44 (2012) 1338-1345.

[3] M. Bani-Hani, R. Abd-Allah, L. El-Khouri, Archeaometallurgical finds from Barsinia, Northern Jordan: Microstructural characterization and conservation treatment, J. Cult. Herit. 13 (2012) 314-325. 

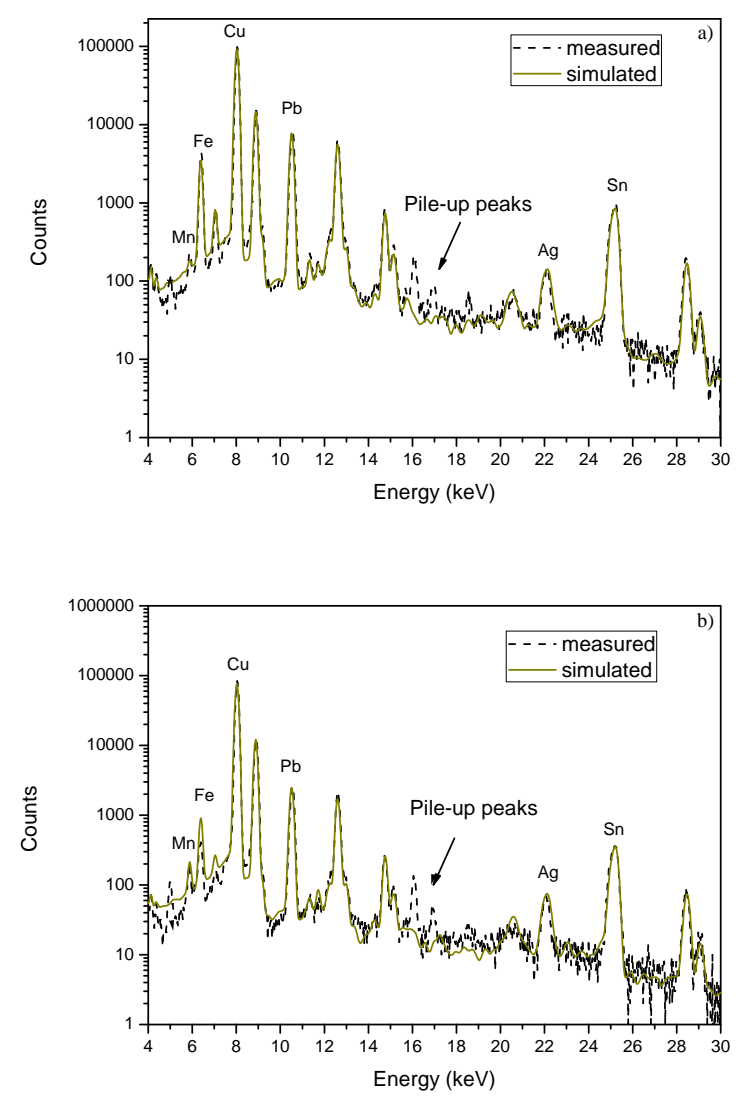

Figure 4: Measured and MC simulated EDXRF spectra obtained a) before and $b$ ) after patina removal.

[4] E. Figueiredo, M. F. Arajo, R. J. Silva, R. Vilaa, Characterisation of a proto-historic bronze collection by micro-EDXRF, Nucl. Instrum. Meth. B 296 (2013) 26-31.

[5] P. Valerio, R. Silva, M. Araajo, A. Soares, L. Barros, A multianalytical approach to study the Phoenician bronze technology in the Iberian Peninsula-A view from Quinta do Almaraz, Mater. Charact. 67 (2012) 74-82.

[6] M. F. Guerra, Analysis of archaeological metals. the place of $\mathrm{XRF}$ and PIXE in the determination of technology and provenance, X-Ray Spectrom. 27 (1998) 73-80.

[7] N. Schiavon, A. Celauro, M. Manso, A. Brunetti, F. Susanna, Iron-age bronze statuettes in southern Portugal: combining archaeological data with EDXRF and BSEM+EDS to assess provenance and production technology, Appl. Phys. A 113 (2013) 865-875.

[8] M. Guerra, S. Longelin, S. Pessanha, M. Manso, M. L. Carvalho, Development of a combined portable Raman and XRF spectrometer for in situ analysis, Rev. Sci. Instrum. (2014) (accepted).

[9] R. Cesareo, A. Brunetti, R. D’Oriano, A. Canu, G. M. Demontis, A. Celauro, A Roman bronze statuette with gilded silver mask from sardinia: an EDXRF study, Appl. Phys. A-Mater 113 (2013) 905-910.

[10] C. Bottaini, J. Mirao, M. Figuereido, A. Candeias, A. Brunetti,
N. Schiavon, Energy dispersive x-ray fluorescence spectroscopy/monte carlo simulation approach for the nondestructive analysis of corrosion patina-bearing alloys in archaeological bronzes: The case of the bowl from the fareleira 3 site (vidigueira, south portugal), Spectrochimica Acta Part B: Atomic Spectroscopy 103-104 (0) (2015) 9-13.

[11] U. Bottigli, A. Brunetti, B. Golosio, P. Oliva, S. Stumbo, L. Vincze, P. Randaccio, P. Bleuet, A. Simionovici, A. Somogyi, Voxel-based Monte Carlo simulation of X-ray imaging and spectroscopy experiments, Spectrochim. Acta B 59 (2004) 1747-1754.

[12] B. Golosio, T. Schoonjans, A. Brunetti, P. Oliva, G. L. Masala, Monte Carlo simulation of X-ray imaging and spectroscopy experiments using quadric geometry and variance reduction techniques, Comput. Phys. Commun. 185 (2014) 1044-1052.

[13] A. M. Arruda, Práticas e rituais no sul de Portugal durante a proto-história, in: Actas do $3^{\circ}$ Congresso de Arqueologia Peninsular, 2000.

[14] A. M. Arruda, Necrópoles proto-históricas do sul de Portugal, in: El Mundo Funerário. Actas del III Seminario Internacional sobre temas Fenícios, 2004.

[15] G. D. Smith, R. J. Clark, Raman microscopy in archaeological science, J. Archaeol. Sci. 31 (2004) 1137-1160.

[16] M. Bouchard, D. Smith, Catalogue of 45 reference Raman spectra of minerals concerning research in art history or archaeology, especially on corroded metals and coloured glass, Spectrochim. Acta A 59 (2003) 2247-2266.

[17] T. Schoonjans, V. A. Sol, L. Vincze, M. S. del Rio, K. Appel, C. Ferrero, A general monte carlo simulation of energydispersive $\mathrm{x}$-ray fluorescence spectrometers $\phi$ part 6. quantification through iterative simulations, Spectrochimica Acta Part B: Atomic Spectroscopy 82 (0) (2013) 36 - 41

[18] T. Schoonjans, A. Brunetti, B. Golosio, M. S. del Rio, V. A. Sole, C. Ferrero, L. Vincze, The xraylib library for X-ray-matter interactions. Recent developments, Spectrochim. B 66 (2011) 776-784.

[19] A. Brunetti, B. Golosio, A new Monte Carlo code for simulation of the effect of irregular surfaces on X-ray spectra, Spectrochim. Acta B 94-95 (2014) 58-62.

[20] M. Serghini-Idrissi, M. Bernard, F. Harrif, S. Joiret, K. Rahmouni, A. Srhiri, H. Takenouti, V. Vivier, M. Ziani, Electrochemical and spectroscopic characterizations of patinas formed on an archaeological bronze coin, Electrochim. Acta 50 (2005) 4699-4709. 